\title{
Amplitude and frequency variability of pulsating stars
}

\author{
G. Handler \\ Institut für Astronomie, Türkenschanzstrasse 17, 1180 Vienna, Austria
}

\begin{abstract}
We review the observations of amplitude and frequency variability of pulsating stars and interpretations thereof. Most of these changes do not seem to be connected to effects of global stellar evolution, and different classes of pulsating star show different behaviour. It is attempted to single out objects for which evolutionary period changes can be measured, and some suggestions that may help the understanding of non-evolutionary amplitude and frequency variations are made.
\end{abstract}

\section{Introduction}

Many regularly pulsating stars change their pulsational frequencies and amplitudes over time. The time scales involved are often shorter than those connected with stellar evolution. The question now arises, what is the reason for those variations and (where) can we use period changes to study stellar evolution?

\section{Amplitude variability}

The Blazhko effect is the most widely known case of amplitude variability, occurring in about one fourth of all RRab stars and in some RRc stars. The light curves of these pulsators are modulated in amplitude on time scales of a few weeks to a few months, and the most promising explanation for the Blazhko effect to date is beating of the high-amplitude radial modes with some nonradial pulsation modes. We refer to Kolenberg (these proceedings) for a review.

Some other explanations for the occurrence of amplitude variations would be pulsational damping if a star is about to leave its instability strip (e.g. Dall et al. 2003), resonant mode interaction (Moskalik 1985), or the excitation and damping of new pulsation modes as the star evolves. All these mechanisms would create a modulation of the intrinsic amplitude of the stellar pulsation modes, whereas others, like the Blazhko effect, or precession of the stellar pulsation axis to the line of sight (Balona 1985) modifying the apparent amplitudes of nonradial modes, only affect the apparent amplitudes of a star.

What is required to understand amplitude variability of pulsating stars? The different physical mechanisms mentioned above would operate on different time scales. Resonant mode interaction or excitation of new modes would modulate the amplitudes on a time scale consistent with the inverse growth rate of a given mode, beating of close frequencies produces a variation with the inverse frequency difference and a predictable phase behaviour, precession would also yield predictable changes, and other mechanisms may introduce rather stochastic modulations. 
The solution would then lie within long-term monitoring with good time coverage of several selected stars, to follow the amplitude (and phase) modulations over several cycles (if any). Unfortunately, such a project seems rather difficult to justify to time allocation committees as the required extent of the observations and expected scientific results are hard to predict.

\section{Period changes}

The study of pulsational period changes appears more fruitful, in particular if they would result in measurement of evolutionary period changes. In the following, we will discuss for which classes of pulsating star determinations for evolutionary period changes seem possible.

\section{$\delta$ Cephei stars}

The rates of period change measured for Cepheids are roughly consistent with evolutionary effects, although some disagreement was noted (see Pietrukowicz 2003 and references therein): besides some unusual objects, Cepheids with periods longer than 10 days show period changes that are slower than predicted by stellar evolutionary models. Pietrukowicz (2003) suggests that this may be due to the stars burning a larger fraction of their Helium than predicted by model calculations.

\section{RR Lyrae stars}

The situation for RR Lyrae stars appears different. For instance, Paparó et al.'s (1998) study of $R_{\mathrm{d}}$ variables in $\mathrm{M} 15$ resulted in a variety of observed behaviour. Some stars even exhibit period change rates with different signs for the fundamental mode and for the first overtone. It therefore seems that period changes of most RR Lyrae variables are not of evolutionary origin.

\section{$\delta$ Scuti stars}

Breger \& Pamyatnykh (1998) have extensively reviewed the period change behaviour of the $\delta$ Scuti stars. They find no evidence that evolutionary period changes were observed so far, but pointed out that pre-main sequence objects may offer good chances for such a determination. Very recently, Handler et al. (2004) measured the rates of period change for the three radial modes of an evolved massive star just crossing the $\delta$ Scuti instability strip, V823 Cas, and found them to be consistent with those predicted from stellar evolution.

roAp stars

The well-studied members of this group show large, sometimes cyclic, period changes, which are not compatible with evolutionary effects, but are probably dominated by magnetic variations. See Kurtz et al. (1997) for details.

\section{$\beta$ Cephei stars}

The period changes of the $\beta$ Cephei stars seem to be similar to those of the $\delta$ Scuti stars, and have been reviewed by Jerzykiewicz \& Pigulski (1998). In many cases, the observed period changes are too large to be compatible with effects from stellar evolution only. It is interesting to note that $\beta$ Cephei stars tend to pulsate in the same modes as the $\delta$ Scuti stars. 
Pulsating white dwarf stars

The period change behaviour of the pulsating white dwarf stars is extremely diverse. However, it can be noted that the shortest periods of these stars change consistently with stellar evolution (e.g. see Kepler et al. 2000), whereas the longer-period variables can show gross frequency changes (e.g. Kleinman et al. 1998). Interestingly, period changes of some of the hottest pulsating white dwarfs could be used to infer the neutrino cooling rate (O'Brien \& Kawaler 2000).

\section{Discussion}

Which stars are most promising for determining evolutionary period changes? Unfortunately, not many seem to be. In our view, only the hottest DA and DB as well as cool DO white dwarfs and short-period Cepheids offer good prospects for such measurements. Some RR Lyrae and $\beta$ Cephei stars as well some more "exotic" cases like evolved massive $\delta$ Scuti stars or pre-main sequence objects may be suitable as well. The case of the pulsating sdB stars, discovered only a few years ago, still remains to be examined when measurements over a longer time baseline become available.

What happens in the stars that show non-evolutionary period changes? It is clear that something in the pulsation cavity must change to modify the mode periods. In this context it is important to remember that nonradial modes are often "weighted" in the stellar interior, i.e. their kinetic energy is dominated by a certain part of the star. For instance, the highovertone gravity modes of pulsating white dwarf stars are mostly confined to regions close to the stellar surface, and hence it requires less energy to modify their cavity than that of low-overtone modes that penetrate in the deep interior - and this is exactly what's observed: the low-overtone modes are much more stable in time.

It would be interesting if observations of consistent behaviour in the period changes of and between other groups of pulsating star could be made and connected. After all, the different classes of pulsator still obey the same basic physics. Consequently, some similarities between them should be expected.

Acknowledgments. This work has been supported by the Austrian Fonds zur Förderung der wissenschaftlichen Forschung under grant R12-N02.

\section{References}

Balona, L. A., 1985, MNRAS 217, 17P

Breger, M., Pamyatnykh, A. A., 1998, A\&A 332, 958

Dall, T. H., Handler, G. Moalusi, M. B., Frandsen, S., 2003, A\&A, in press

Handler, G., Rodríguez, E., Ezhkova, O. V., Costa, V., Krzesinski, J., Antipin, S. V., 2004, MNRAS, in preparation

Jerzykiewicz, M., Pigulski, A., 1998, in A Half Century of Stellar Pulsation Interpretations: A Tribute to Arthur N. Cox, ed. P. A. Bradley \& J. A. Guzik, ASP Conf. Ser. 135, p. 43

Kepler, S. O., Mukadam, A., Winget, D. E., Nather, R. E., Metcalfe, T. S., Reed, M. D., Kawaler, S. D., Bradley, P. A., 2000, ApJ 534, L185

Kleinman, S. J., et al., 1998, ApJ 495, 424

Kurtz, D. W., van Wyk, F., Roberts, G., Marang, F., Handler, G., Medupe, R., Kilkenny, D., 1997, MNRAS 287, 69

Moskalik, P., 1985, Acta Astron. 35, 229

O'Brien, M. S., Kawaler, S. D., 2000, ApJ 539, 372

Paparó, M., Saad, S. M., Szeidl, B., et al., 1998, A\&A 332, 102

Pietrukowicz, P., 2003, Acta Astron. 53, 63 